Pacific Journal of Mathematics

HOMOLOGICAL DIMENSION AND SPLITTING TORSION 


\title{
HOMOLOGICAL DIMENSION AND SPLITTING TORSION THEORIES
}

\author{
MARK L. TEPLY
}

The concept of a torsion theory $(\mathscr{T}, \mathscr{F})$ for left $R$ modules has been defined by S. E. Dickson. A torsion theory is called splitting if it has the property that the torsion submodule of every left $R$-module is a direct summand. Under restrictive hypotheses on the ring $R$, several specific splitting theories have previously been examined. This paper continues the investigation to more general classes of torsion theories. In the first section, comparisons are made between injective modules and torsion modules for a splitting theory, and the following results are obtained: (1) A torsion class $\mathscr{T}$ is closed under taking injective envelopes if and only if the maximal $\mathscr{T}$-torsion submodule of an injective module is injective. (2) If $(\mathscr{T}, \mathscr{F})$ is splitting and $R \in \mathscr{F}$, then inj $\operatorname{dim}(T) \leqq 1$ for all $T \in \mathscr{T}$. (3) If $(\mathscr{T}, \mathscr{F})$ is splitting and hereditary and if $R \in \mathscr{F}$, then every homomorphic image of a $\mathscr{T}$-torsion injective module is injective. In $\S 2$ it is shown that rings $R$, for which $R$ has zero singular ideal and Goldie's torsion theory is splitting, have the property: $1 . \operatorname{g1} 1 \operatorname{dim} R \leqq 2$. It is shown that the relative homological dimension arising from a hereditary torsion theory often gives information about splitting, especially when this dimension is zero. In the final sections, the zero-dimensionality of a hereditary torsion theory is discussed and related to results of J. P. Jans. The rings, all of whose hereditary torsion theories have dimension zero, are characterized as direct sums of finitely many right perfect rings, each of which has a unique maximal ideal.

In this paper, all rings $R$ have identity, and all modules are unitary left $R$-modules. The category of left $R$-modules is denoted by $R \mathscr{l}$.

A torsion theory of modules is a pair $(\mathscr{T}, \mathscr{F})$ of subclasses of R $\mathscr{A l}$ satisfying:

(1) $\mathscr{T} \cap \mathscr{F}=\{0\}$.

(2) $B \subseteq A$ and $A \in \mathscr{T}$ implies $A / B \in \mathscr{T}$.

(3) $B \leqq A$ and $A \in \mathscr{F}$ implies $B \in \mathscr{F}$.

(4) For each $A \in_{R} \mathscr{H}$, there exists a (necessarily unique) exact sequence

$$
0 \longrightarrow T \longrightarrow A \longrightarrow F
$$

such that $T \in \mathscr{T}$ and $F \in \mathscr{F}$.

For this definition and the following results, the reader is referred 
to $[5]$.

Let $(\mathscr{T}, \mathscr{F})$ be a torsion theory for ${ }_{R} \mathscr{M}$. Modules in $\mathscr{T}$ are called torsion, and those in $\mathscr{F}$ are called torsionfree. Each $A \in_{R} \mathscr{C l}$ has a unique maximal torsion submodule, denoted by $\mathscr{T}(A) . \mathscr{T}$ is closed under taking direct sums, and $\mathscr{F}$ is closed under taking direct products. $\mathscr{T}=\left\{T \in_{R} \mathscr{M} \mid \operatorname{Hom}_{R}(T, F)=0\right.$ for all $\left.F \in \mathscr{F}\right\}$, and $\mathscr{F}=\left\{F \in_{R} \mathscr{K} \mid \operatorname{Hom}_{R}(T, F)=0\right.$ for all $\left.T \in \mathscr{T}\right\}$. A subclass $\mathscr{C}$ of ${ }_{R} \mathscr{C l}$ is closed under taking extensions if $A, B \in \mathscr{C}$ and $0 \rightarrow A \rightarrow X \rightarrow B \rightarrow 0$ is exact imply $X \in \mathscr{C}$. Both $\mathscr{T}$ and $\mathscr{F}$ are closed under taking extensions. A class $\mathscr{C}$ is closed under taking injective envelopes if $A \in \mathscr{C}$ implies $E(A) \in \mathscr{C}$, where $E(A)$ denotes the injective envelope of $A$. $\mathscr{T}$ is closed under taking submodules if and only if $\mathscr{F}$ is closed under taking injective envelopes. When $(\mathscr{T}, \mathscr{F})$ has this property, then $(\mathscr{T}, \mathscr{F})$ is called a hereditary torsion theory. In this case $\mathscr{T}$ is also a class of negligible modules in the sense of P. Gabriel [10], and hence there is a topologizing and idempotent filter $F(\mathscr{T})$ of left ideals associated with $\mathscr{T}$. For results concerning these filters, the reader is referred to [10] or [15].

For convenience $\operatorname{Ext}_{R}^{n}(A, B)$ will be written as $\operatorname{Ext}^{n}(A, B)$ throughout this paper. The following notations concerning homological dimensions are used for the ring $R$ and the $R$-module $M$ :

$$
\begin{aligned}
\operatorname{inj} \operatorname{dim}(M) & =\inf \left\{n \mid \operatorname{Ext}^{n+1}(-, M)=0\right\} \\
\text { h. } \operatorname{dim}(M) & =\inf \left\{n \mid \operatorname{Ext}^{n+1}(M,-)=0\right\} \\
\text { 1. gl. } \operatorname{dim} R & =\inf \left\{\text { h. } \operatorname{dim}(M) \mid M \in_{R} \mathscr{C l}\right\} .
\end{aligned}
$$

1. Injectives and splitting. Let $(\mathscr{T}, \mathscr{F})$ be a splitting torsion theory for ${ }_{R} \mathscr{C l}$, i.e., $\mathscr{T}(M)$ is a summand of each $M \in_{R} \mathscr{M}$. Since an injective module is always a summand of any module containing it, it is natural to wonder how much a module in $\mathscr{T}$ must "resemble" an injective module. The first lemma examines the case of the maximal torsion submodule of an injective module. It shows that the splitting of $(\mathscr{T}, \mathscr{F})$ implies that $\mathscr{T}$ is closed under taking injective envelopes.

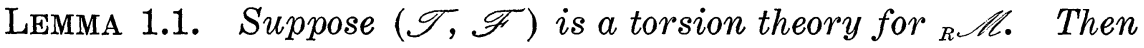
$\mathscr{T}$ is closed under injective envelopes if and only if $\mathscr{T}(A)$ is injective for each injective module $A \in_{R} \mathscr{M}$.

Proof. $(\Rightarrow)$ : Let $A$ be injective. Then $E(\mathscr{T}(A)) \in \mathscr{T}$ by hypothesis. But then $E(\mathscr{T}(A)) / \mathscr{T}(A) \in \mathscr{T}$ and

$$
E(\mathscr{T}(A)) / \mathscr{T}(A) \leqq A / \mathscr{T}(A) \in \mathscr{F} .
$$

Hence $E(\mathscr{T}(A)) / \mathscr{T}(A) \in \mathscr{T} \cap \mathscr{F}=\{0\}$. 
$(\Longleftrightarrow)$ : Let $T \in \mathscr{T}$. By hypothesis, $E(T)=\mathscr{T}(E(T)) \oplus F$, where $F \in \mathscr{F}$. Since $\mathscr{T}(E(T))+T \in \mathscr{T}$ is contained in $E(T)$, then $T \leqq$ $\mathscr{T}(E(T))$, and hence $F=0$.

The following lemma is clear:

LEMMA 1.2. The following are equivalent for a torsion theory $(\mathscr{T}, \mathscr{F})$ for ${ }_{R} \mathscr{l l}$.

(1) $(\mathscr{T}, \mathscr{F})$ is splitting.

(2) $\operatorname{Ext}(F, T)=0$ for all $F \in \mathscr{F}, T \in \mathscr{T}$.

THEOREM 1.3. Let $(\mathscr{T}, \mathscr{F})$ be a splitting torsion theory for RMll. If $R \in \mathscr{F}$, then $\operatorname{inj} \operatorname{dim}(T) \leqq 1$ for all $T \in \mathscr{T}$.

Proof. Since $R \in \mathscr{F}$, every submodule of a free $R$-module is in $\mathscr{F}$. So for each $M \in_{R} / l$, there is an exact sequence

$$
0 \longrightarrow K \longrightarrow F \longrightarrow M \longrightarrow 0
$$

with $F$ projective and $K, F \in \mathscr{F}$. Hence by Lemma 1.2 , the exact sequence

$$
\operatorname{Ext}(K, T) \longrightarrow \operatorname{Ext}^{2}(M, T) \longrightarrow \operatorname{Ext}^{2}(F, T)=0
$$

yields $\operatorname{Ext}^{2}(M, T)=0$ for all $T \in \mathscr{T}$.

Now suppose for induction that $\operatorname{Ext}^{n}(M, T)=0$ for all $T \in \mathscr{T}$. If $T \in \mathscr{T}$, then $E(T) \in \mathscr{T}$ by Lemma 1.1, and hence $E(T) / T \in \mathscr{T}$. So, by the induction hypothesis, the exact sequence

$$
\operatorname{Ext}^{n}(M, E(T) / T) \longrightarrow \operatorname{Ext}^{n+1}(M, T) \longrightarrow \operatorname{Ext}^{n+1}(M, E(T))=0
$$

yields $\operatorname{Ext}^{n+1}(M, T)=0$ for all $T \in \mathscr{T}$.

Hence the result follows by induction.

Corollary 1.4. Let $(\mathscr{T}, \mathscr{F})$ be a splitting torsion theory for ${ }_{R \sim} / l$. Let $A$ be an injective module and $f$ a homomorphism of $A$. If $R \in \mathscr{F}$ and if the kernel of $f$ is in $\mathscr{T}$, then the image of $f$ is injective.

Proof. Let $K$ be the kernel of $f$, and let $I$ be the image of $f$. Then Theorem 1.3 yields the following exact sequence for any $M \in_{R} \mathscr{l}$ :

$$
0=\operatorname{Ext}^{1}(M, A) \longrightarrow \operatorname{Ext}^{1}(M, I) \longrightarrow \operatorname{Ext}^{2}(M, K)=0 \text {. }
$$

Hence $\operatorname{Ext}^{1}(M, I)=0$ by exactness, and so $I$ is injective.

The following result is the special case of Corollary 1.4 for a hereditary torsion theory. 
COROLlaRY 1.5. Let $(\mathscr{T} ; \mathscr{F})$ be a splitting hereditary torsion theory for ${ }_{R} \mathscr{C l}$. If $R \in \mathscr{F}$, then every homomorphic image of a torsion injective module is injective.

2. The Goldie theory. A submodule $A \subseteq M$ is said to be essential in $M$ if $A \cap B \neq 0$ for every nonzero submodule $B$ of $M$. The singular submodule of $M \in_{R} \mathscr{C l}$ is $Z(M)=\{x \in M \mid(0: x)$ is essential in $R$. If $Z(M)=0$, then $M$ is called nonsingular.

Goldie's torsion theory $(\mathscr{G}, \mathscr{N})$ is the torsion theory given by $\mathscr{N}=\left\{N \in_{R} \mathscr{M} \mid N\right.$ is nonsingular $\}$ and $\mathscr{G}=\left\{G \in_{R} \mathscr{M} \mid Z(G)\right.$ is essential in $G\} . \quad(\mathscr{G}, \mathscr{N})$ is hereditary and has as its filter $F(\mathscr{G})=\{I \mid I \subseteq$ $J$ essential in $R$, and $(I: x)$ is essential in $R$ for all $x \in J\}$. This is the smallest topologizing and idempotent filter containing the essential left ideals. For other results on $(\mathscr{G}, \mathscr{N})$, the reader is referred to [1], [11] or [14].

V. Cateforis and F. Sandomierski [4] have studied the splitting of $(\mathscr{G}, \mathscr{N})$ for commutative rings with $Z(R)=0 . \quad Z(R)=0$ if and only if $Z(M)=\mathscr{G}(M)$ for all $M \in_{R} \mathscr{l l}$. Hence saying $(\mathscr{G}, \mathscr{N})$ splits and $Z(R)=0$ is equivalent to saying that the singular submodule always splits off. In [4] it is shown that whenever $(\mathscr{G}, \mathscr{N})$ is splitting, $R$ is commutative, and $Z(R)=0$, then $1 . \mathrm{gl} . \operatorname{dim} R \leqq 1$. The results below show that this bound can be kept for modules in $\mathscr{N}$ (i.e., $h \cdot \operatorname{dim}(N) \leqq 1$ for all $N \in \mathscr{N}$ ) when the commutative hypothesis on $R$ is dropped. Moreover, if $(\mathscr{G}, \mathscr{N})$ splits and $Z(R)=0$, then l. gl. $\operatorname{dim} R \leqq 2$.

THEOREM 2.1. If $(\mathscr{G}, \mathscr{N})$ splits and $R \in \mathscr{N}$, then $h \cdot \operatorname{dim}(N) \leqq 1$ for all $N \in \mathscr{N}$.

Proof. Let $N, F \in \mathscr{N}$. Then $E(N) / N \in \mathscr{G}$, so that

$$
\operatorname{Ext}(F, E(N) / N)=0
$$

by Lemma 1.2. Then the exact sequence

$$
0=\operatorname{Ext}^{1}(F, E(N) / N) \longrightarrow \operatorname{Ext}^{2}(F, N) \longrightarrow \operatorname{Ext}^{2}(F, E(N))=0
$$

yields $\operatorname{Ext}^{2}(F, N)=0$ for all $F, N \in \mathscr{N}$. By Theorem 1.3,

$$
\operatorname{Ext}^{n}(F, E(N) / N)=0
$$

for all $n \geqq 2$. So the exact sequence

$$
0=\operatorname{Ext}^{n}(F, E(N) / N) \longrightarrow \operatorname{Ext}^{n+1}(F, N) \longrightarrow \operatorname{Ext}^{n+1}(F, E(N))=0
$$

yields $\operatorname{Ext}^{n+1}(F, N)=0$ for all $F, N \in \mathscr{N}$ and all $n \geqq 2$.

Let $M \in_{R} \mathscr{M}$. By splitting $M \cong \mathscr{G}(M) \oplus M / \mathscr{G}(M)$. Hence 


$$
\operatorname{Ext}^{n}(F, M) \cong \operatorname{Ext}^{n}(F, \mathscr{G}(M)) \oplus \operatorname{Ext}^{n}(F, M / \mathscr{G}(M))=0
$$

for all $n \geqq 2$ and all $F \in \mathscr{N}$, by Theorem 1.3 and the first part of the proof.

THEOREM 2.2. If $(\mathscr{G}, \mathscr{N})$ splits and $R \in \mathscr{N}$, then l. gl. $\operatorname{dim} R \leqq 2$.

Proof. Let $F \in \mathscr{N}$ and $M \in_{R} \mathscr{M l}$. By Theorem 1.3 there is an exact sequence

$$
0=\operatorname{Ext}^{n-1}(M, E(F) / F) \longrightarrow \operatorname{Ext}^{n}(M, F) \longrightarrow \operatorname{Ext}^{n}(M, E(F))=0
$$

for all $n \geqq 3$. Thus $\operatorname{Ext}^{n}(M, F)=0$ for all $n \geqq 3$.

Let $M, M_{1} \in_{R} \mathscr{C}$. By splitting $M_{1} \cong \mathscr{G}\left(M_{1}\right) \oplus M_{1} / \mathscr{G}\left(M_{1}\right)$. Hence, for $n \geqq 3$,

$$
\operatorname{Ext}^{n}\left(M, M_{1}\right)=\operatorname{Ext}^{n}\left(M, \mathscr{G}\left(M_{1}\right)\right) \oplus \operatorname{Ext}^{n}\left(M, M_{1} / \mathscr{G}\left(M_{1}\right)\right)=0
$$

by Theorem 1.3 and the first part of the proof. Hence 1. gl. $\operatorname{dim} R \leqq 2$.

3. Relative homological algebra. In [6] the right derived functors of a torsion subfunctor of the identity were calculated. This leads to a relativized injective dimension of modules for each hereditary torsion theory, and hence to a global dimension of ${ }_{R} \mathscr{M}$ depending on the hereditary torsion theory $(\mathscr{T}, \mathscr{F}$ ) chosen. This global dimension is denoted by $\mathscr{T}$ gl. $\operatorname{dim} . R$.

In [1] it is shown that if $\mathscr{G}$ gl. $\operatorname{dim} . R=0$, then $(\mathscr{G}, \mathscr{N})$ splits. S. E. Dickson has conjectured [7] that the simple theory $(\mathscr{S}, \mathscr{F})$ (i.e., the torsion theory whose torsion class is the smallest torsion class containing the simple $R$-modules) splits if and only if $\mathscr{S}={ }_{R} \mathscr{C l}$. In this section it is shown that $\mathscr{S}={ }_{R} \mathscr{C}$ if and only if $\mathscr{S} \mathrm{gl} \cdot \operatorname{dim} . R=$ 0 . Moreover, for any hereditary torsion theory $(\mathscr{T}, \mathscr{F})$, Theorem 3.1 below shows that $\mathscr{T} \mathrm{gl} \cdot \operatorname{dim} . R=0$ if and only if $\mathscr{F}$ is a TTF class in the sense of [13], i.e., a class closed under taking submodules, factor modules, direct products, and extensions.

The first right derived functor of $A \in_{R} \sim \mathcal{C}$ relative to the hereditary torsion theory $(\mathscr{T}, \mathscr{F})$ is

$$
R_{\mathscr{T}}(A)=\mathscr{T}(E(A) / A) / \frac{\mathscr{T}(E(A))+A}{A} .
$$

Then $\mathscr{T}$ gl. $\operatorname{dim} . R=0$ if and only if $R_{\mathscr{T}}(A)=0$ for all $A \in_{R} / l$.

Following [1], a module $F \in \mathscr{F}$ called $\mathscr{T}$-absolutely pure (relative to the hereditary torsion theory $(\mathscr{F}, \mathscr{F})$ ) if $L \supseteqq F$ and $L \in \mathscr{F}$ imply $L / F \in \mathscr{F}$. [1], Proposition 1.4 states that $F \in \mathscr{F}$ is $\mathscr{T}$-absolutely pure if and only if $E(F) / F \in \mathscr{F}$. 
THEOREM 3.1. For a hereditary torsion theory $(\mathscr{T}, \mathscr{F})$, the following are equivalent:

(1) $T: M \rightarrow \mathscr{T}(M) \forall M \in_{R} \mathscr{C l}$ is an exact functor.

(2) Every $F \in \mathscr{F}$ is $\mathscr{T}$-absolutely pure.

(3) $\mathscr{F}$ is closed under taking homomorphic images.

(4) $\mathscr{T}$ gl. $\operatorname{dim} . R=0$.

Proof. $(1) \Rightarrow(2)$ : Let $F, L \in \mathscr{F}$ and $L \supseteq F$. Then apply the exact functor $T$ to the exact sequence $0 \rightarrow F \rightarrow L \rightarrow L / F \rightarrow 0$ to get $0 \rightarrow \mathscr{T}(F) \rightarrow \mathscr{T}(L) \rightarrow \mathscr{T}(L / F) \rightarrow 0$. Since $L \in \mathscr{F}$, then $\mathscr{T}(L / F)=0$ by exactness, and hence $L / F \in \mathscr{F}$. Thus $F$ is $\mathscr{T}$-absolutely pure.

$(2) \Longrightarrow(3)$ : Let $f: F \rightarrow M$ be an epimorphism of $F \in \mathscr{F}$, and let $K$ be the kernel of $f$. Since $\mathscr{F}$ is closed under taking submodules, $K \in \mathscr{F}$, and hence $M \cong F / K \in \mathscr{F}$ by $(2)$.

$(3) \Rightarrow(4)$ : For any $M \in_{R} \mathscr{C l}$, the exact sequence

$$
0 \longrightarrow \mathscr{T}(M) \longrightarrow M \longrightarrow M / \mathscr{T}(M) \longrightarrow 0
$$

induces the exact sequence

$$
R_{\mathscr{T}}(\mathscr{T}(M)) \longrightarrow R_{\mathscr{T}}(M) \longrightarrow R_{\mathcal{Y}}(M / \mathscr{T}(M)) .
$$

By [6], Lemma 2, $R,(\mathscr{T}(M))=0$. Hence it is sufficient to show that $R_{\mathscr{\sigma}}(F)=0$ for all $F \in \mathscr{F}$. Since $\mathscr{F}$ is closed under injective envelopes, $\mathscr{T}(E(F))=0$ for all $F \in \mathscr{F}$. Hence the formula for $R_{-}(F)$ reduces to $\mathscr{T}(E(F) / F)$ whenever $F \in \mathscr{F}$. But (3) and $E(F) \in \mathscr{F}$ imply $E(F) / F \in \mathscr{F}$, and hence $R_{J}(F)=\mathscr{T}(E(F) / F)=0$.

$(4) \Rightarrow(1)$ : This is clear since $T$ is always left exact.

The simple torsion theory $(\mathscr{S}, \mathscr{F})$ has $\mathscr{S}$ defined [5] by $T \in \mathscr{S}$ if and only if every nonzero homomorphic image of $T$ has nonzero socle. Then $\mathscr{F}$ corresponding to $\mathscr{S}$ is the class of modules with zero socle.

COROLlary 3.2. The following are equivalent:

(1) $\mathscr{S}$ gl. $\operatorname{dim} R=0$.

(2) Nonzero modules have nonzero socles.

Proof. $\quad(1) \Rightarrow(2)$ : Suppose $R \notin \mathscr{S}$, so that $\mathscr{S}(R)$ is a proper ideal of $R$. Let $M$ be a maximal left ideal of $R$ containing $\mathscr{S}(R)$. Then $R / M \in \mathscr{S}$ is a homomorphic image of $R / \mathscr{S}(R) \in \mathscr{F}$. But (1) and Theorem 3.1 (3) yield $R / M \in \mathscr{F}$, which contradicts $\mathscr{S} \cap \mathscr{F}=0$. Hence $R \in \mathscr{S}$, and so $\mathscr{S}={ }_{R} \mathscr{C l}$, i.e., (2) holds.

$(2) \Rightarrow(1):$ By $(2), \mathscr{S}={ }_{R} \mathscr{C l}$ and hence $\mathscr{F}=\{0\}$. Thus $\mathscr{F}$ is trivially closed under homomorphic images, and hence (1) follows from Theorem 3.1. 
Let $\mathscr{T}_{S}$ denote the smallest torsion class containing the simple $R$-module $S$. If each $T \in \mathscr{S} \subseteq{ }_{R} \mathscr{M}$ can be written as

$$
T=\bigoplus \sum_{S \in \mathscr{C}} \mathscr{T}_{S}(T),
$$

where $\mathscr{C}$ is a set of nonisomorphic simple $R$-modules, then $R$ is said to have primary decomposition (PD) for $\mathscr{S}$. For further results on (PD), the reader is referred to [5] and [9].

In order to characterize rings for which every hereditary torsion theory has dimension zero, the following result of $\mathrm{H}$. Bass [2] is needed:

THEOREM P. The following are equivalent:

(1) $R$ is right perfect.

(2) $R / J(R)$ is semi-simple Artinian and $J(R)$ is right T-nilpotent, where $J(R)$ denotes the Jacobson radical of $R$.

(3) $R$ contains no infinite sets of orthogonal idempotents and nonzero left modules have nonzero socles.

THEOREM 3.3. Every hereditary torsion theory $(\mathscr{T}, \mathscr{F})$ for ${ }_{R} \mathscr{t l}$ has $\mathscr{T} \mathrm{gl} \cdot \operatorname{dim} R=0$ if and only if $R$ is the direct sum of finitely many right perfect rings, each of which has a unique maximal twosided ideal.

Proof. $(\Longrightarrow)$ : By $\mathscr{S}$ gl. $\operatorname{dim} R=0$ and Corollary 3.2, nonzero modules have nonzero socles. From $\mathscr{T}_{S}$ gl. $\operatorname{dim} R=0$, Theorem 3.1, and [5], Theorem 5.3, it follows that $R$ has (PD). Since each $\mathscr{T}_{S}(R)$ is a two sided ideal, then $R=R_{1}+R_{2}+\cdots+R_{n}$ (ring direct sum), where each $R_{i}=\mathscr{T}_{S}(R)$ for some simple module $S$. Then nonzero left $R_{i}$-modules have nonzero socles, and hence $J\left(R_{i}\right)$, the Jacobson radical of $R$, is right $T$-nilpotent by an argument of H. Bass [2].

It remains to show that $R_{i} / J\left(R_{i}\right)$ is a simple Artinian ring; for then the required properties of $R_{i}$ follow from Theorem P. Let $B$ be the inverse image in $R_{i}$ of Soc $\left(R_{i} / J\left(R_{i}\right)\right)$; then $B$ is a two-sided ideal of $R_{i}$. If $B \neq R_{i}$ and $M$ is any maximal left ideal of $R_{i}$ containing $B$, then the following property holds: $R_{i} / M \cong R_{i} / M^{\prime}$ implies $M^{\prime} \supseteqq B$. Since nonzero $R_{i}$-modules have nonzero socles, then $B \neq J\left(R_{i}\right)$. So since $J\left(R_{i}\right)$ is the intersection of maximal left ideals of $R_{i}$, it follows that there exists a maximal left ideal $M_{1}$ such that $M_{1} \nsupseteq B$ and hence $R_{i} / M \not R_{i} / M_{1}$. This contradicts the fact that $R_{i}$ has only one simple $R_{i}$-module (up to isomorphism). Hence $B=R$, i.e., $R_{i} / J\left(R_{i}\right)=\operatorname{Soc}\left(R_{i} / J\left(R_{i}\right)\right)$. Hence $R_{i} / J\left(R_{i}\right)$ is semi-simple Artinian. Since $R_{i}$ has only one simple $R_{i}$-module up to isomorphism, then $R_{i} / J\left(R_{i}\right)$ is a simple ring. 
$(\Longleftrightarrow)$ : Let $R=R_{1}+R_{2}+\cdots+R_{n}$ (ring direct sum), where each $R_{i}$ is a right perfect ring with a unique maximal ideal. Then from Theorem $P$ it follows that nonzero modules have nonzero socles. So for any hereditary torsion theory $(\mathscr{T}, \mathscr{F})$ either $R_{i} \in \mathscr{T}$ or $R_{i} \in \mathscr{F}$ for $i=1,2, \cdots, n$. Then it is not hard to see that $\mathscr{F}$ is closed under homomorphic images, and hence $\mathscr{T} \mathrm{gl} \cdot \operatorname{dim} R=0$ by Theorem 3.1.

A torsion theory $(\mathscr{T}, \mathscr{F})$ for ${ }_{R} \mathscr{C l}$ is said to be of simple type if it is hereditary and nonzero modules in $\mathscr{T}$ have nonzero socles. Then $(\mathscr{T}, \mathscr{F})$ is of simple type if and only if $\mathscr{T}$ is the smallest torsion class containing a given set of simple modules.

CoRollary 3.4. Suppose every hereditary torsion theory $(\mathscr{T}, \mathscr{F})$ for ${ }_{R} \mathscr{C l}$ has $\mathscr{T} \mathrm{gl} \cdot \operatorname{dim} R=0$. Then the following are equivalent:

(1) Every torsion theory for ${ }_{R} \mathscr{M}$ is of simple type.

(2) $J(R)$ is left T-nilpotent.

(3) Nonzero left $R$-modules have maximal submodules.

Proof. (2) $\Leftrightarrow(3)$ is immediate from [12], Lemma 1 and Theorem 3.3. $(1) \Longrightarrow(3)$ : Let $0 \neq A \in_{R} \mathscr{C l}$ be a module with no maximal submodule. Define $\mathscr{F}$ by $\mathscr{F}=\left\{X \in_{R} \mathscr{C l} \mid \operatorname{Hom}(A, X)=0\right\}$. It is easily checked that $\mathscr{F}$ is closed under taking submodules, extensions, and direct products; hence $\mathscr{F}$ is a torsionfree class by [5], Theorem 2.3. Since all the simple left $R$-modules are in $\mathscr{F}$, this contradicts (1).

$(3) \Rightarrow(1)$ : From Theorem 3.3 it follows that nonzero left modules have nonzero socles. Let $(\mathscr{T}, \mathscr{F}$ ) be a torsion theory. It is sufficient to prove that for each $M \in \mathscr{T}$, $\operatorname{Soc}(M) \in \mathscr{T}$. If $S$ is a simple submodule of $M \in \mathscr{T}$, then choose $N$ maximal in the properties $N \subseteq M$ and $N \cap S=0$. Then $S$ is isomorphic to an essential submodule of $M / N \in \mathscr{T}$. Since $R$ has (PD), it follows that $M / N \in \mathscr{T}_{s}$, where $\mathscr{T}_{S}$ is the smallest torsion class containing $S$. Thus every maximal submodule $T / N$ of $M / N$ has the property $(M / N) /(T / N) \cong S$. (Such maximal submodules exist by (3).) Thus $M / N \in \mathscr{T}$ implies $S \in \mathscr{T}$.

CoRollary 3.5. Let $R$ be commutative. Then the following are equivalent:

(1) Every hereditary torsion theory $(\mathscr{T}, \mathscr{F}$ ) has $\mathscr{T}$ gl. dim. $R=0$.

(2) $R$ is a direct sum of finitely many local perfect rings.

(3) $h . \operatorname{dim}(M)=0$ or $\infty$ for each $M \in_{R} \mathscr{M l}$.

(4) Every torsion theory for ${ }_{R} \mathscr{C l}$ is splitting.

(5) $R$ has (PD) and $(\mathscr{S}, \mathscr{F})$ is splitting.

Proof. $\quad(1) \Leftrightarrow(2)$ is Theorem $3.3 ;(2) \Leftrightarrow(3)$ is a result of $\mathrm{I}$. Kaplansky (see [2]); and $(2) \Leftrightarrow(5)$ is [9], Theorem 5.4. 
(1) and $(2) \Rightarrow(4)$ : By Corollary 3.4, every torsion theory is of simple type. (PD) follows from (2), and hence every torsion theory splits.

$(4) \Rightarrow(5)$ : Suppose (PD) does not hold. Then there exists nonzero $M \in_{R} \mathscr{C l}$ such that $\mathscr{S}(M) \neq \oplus \sum_{S \in A} \mathscr{T}_{S}(M)$, where $A$ is a representative set of nonisomorphic simple modules. Let

$$
S^{\prime} \cong N / \sum_{S \in A} \mathscr{T}_{S}(M) \subseteq M / \sum_{S \in A} \mathscr{T}_{S}(M)
$$

The $\mathscr{T}_{S^{\prime}}$-torsion part of $N$ is $\mathscr{T}_{S^{\prime}}(M)$, by splitting $N=\mathscr{T}_{S^{\prime}}(M) \oplus K$ and $\mathscr{T}_{S}(K)=K \cap \mathscr{T}_{S}(M)=\mathscr{T}_{S}(M)$. Since $K \neq \sum_{s \in A} \mathscr{T}_{S}(M)$, then

$$
S^{\prime} \cong K / \sum_{S \in A-\left\{S^{\prime}\right\}} \mathscr{T}_{S}(M) \text {. }
$$

Since the smallest torsion theory containing the set $A-\left\{S^{\prime}\right\}$ splits, then

$$
K \cong\left[\sum_{S \in A-\left\{S^{\prime}\right\}} \mathscr{T}_{S}(M)\right] \oplus S^{\prime}
$$

which is a contradiction to $K \cap \mathscr{T}_{S^{\prime}}(M)=0$.

4. Central splitting. A pair of torsion theories $(\mathscr{C}, \mathscr{T})$, $(\mathscr{T}, \mathscr{F})$ is called a torsion-torsionfree (TTF) theory. In this case $\mathscr{T}$ is both a torsion and a torsionfree class, and hence $\mathscr{T}$ is called a TTF class as in [13]. In $\S 3$ it was pointed out that TTF theories are related to $\mathscr{C} \mathrm{gl} . \operatorname{dim} . R=0$, whenever $(\mathscr{C}, \mathscr{T})$ is hereditary. The splitting of TTF theories is studied in [13], and the following is the main result obtained:

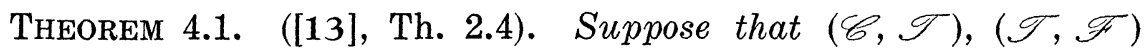
is a TTF theory. Then the following are equivalent:

(1) For all $M \in_{R} \mathscr{M l}, M=\mathscr{C}(M) \oplus \mathscr{T}(M)$.

(2) $R=\mathscr{C}(R)+\mathscr{T}(R)$ (ring direct sum).

(3) $\mathscr{F}=\mathscr{C}$.

(4) $\mathscr{T}(\mathscr{C}(M))=0$ and $\mathscr{C}(M / \mathscr{T}(M))=M / \mathscr{T}(M)$ for all $M \epsilon_{R} \mathscr{M l}$.

The following questions concerning a TTF theory $(\mathscr{C}, \mathscr{T}),(\mathscr{T}, \mathscr{F})$ were raised in a conversation between R. L. Bernhardt and the author: (1) If $(\mathscr{T}, \mathscr{F})$ is splitting, is $(\mathscr{C}, \mathscr{T})$ also splitting? (2) In case $(\mathscr{C}, \mathscr{T})$ is splitting, when does $(\mathscr{C}, \mathscr{T})$ have the special type of splitting described in Theorem 4.1?

Examples are given to show that either one of $(\mathscr{C}, \mathscr{T})$ or $(\mathscr{T}, \mathscr{F})$ may be splitting without the other splitting. Conditions under which the splitting of one implies the splitting of the other are discussed. 
If $(\mathscr{C}, \mathscr{T})$ satisfies the condition described in Theorem 4.1 (1), then $(\mathscr{C}, \mathscr{T})$ will be called central splitting (as in [3]). The following result ([13], Th. 2.1) may be useful to the reader in the sequel: A hereditary torsion theory $(\mathscr{T}, \mathscr{F})$ for ${ }_{R} \mathscr{C l}$ has the property that $\mathscr{T}$ is closed under taking direct products if and only if the filter $F(\mathscr{T})=\{K \mid R / K \in \mathscr{T}, K$ is a left ideal of $R\}$ has a smallest element. $I$. In this case $I=\mathscr{C}(R)$, where $(\mathscr{C}, \mathscr{T})$ is a torsion theory.

EXAMPLE 4.2. $\mathscr{G}$ is a TTF class and $(\mathscr{G}, \mathscr{N})$ is splitting, but $(\mathscr{C}, \mathscr{G})$ is not splitting. Let $K$ be a field and $A$ a countably infinite index set. Let $Q=\prod_{\alpha \in A} K^{(\alpha)}$, where $K^{(\alpha)}=K$. Then let

$$
R=\sum_{\alpha \in A} K^{(\alpha)}+K \cdot 1 \subseteq Q,
$$

where $1 \in Q$. It is shown in [4] that the Goldie torsion theory $(\mathscr{G}, \mathscr{N})$ is splitting. Since $Z(R)=0$, then $F(\mathscr{G})=\left\{R, \sum_{\alpha \in A} K^{(\alpha)}\right\}$, and hence $\mathscr{G}$ is closed under products. Finally, $(\mathscr{C}, \mathscr{G})$ is not splitting since $\mathscr{C}(R)=\sum_{\alpha \in A} K^{(\alpha)}$ is not a summand of $R$.

Before stating the first sufficient condition for the splitting of $(\mathscr{T}, \mathscr{F})$ to imply the splitting of $(\mathscr{C}, \mathscr{T})$, a lemma due to S. E. Dickson is needed. [7], Proposition 1 is a weaker form of this lemma, however, the proofs are almost identical.

LEMMA 4.3. Let $I=\sum_{i=1}^{n} m_{i} R$ be a finitely generated right ideal of $R$. Then the class $\mathscr{D}=\left\{D \in_{R} \mathscr{C l} \mid I D=D\right\}$ is closed under direct products.

Proof. Let $D_{\alpha} \in \mathscr{D}(\alpha \in B)$. If $x \in \prod_{\alpha \in B} D_{\alpha}$, then for each $\alpha \in B$ there are $x_{1}^{(\alpha)}, x_{2}^{(\alpha)}, \cdots, x_{n}^{(\alpha)} \in D_{\alpha}$ such that

$$
x_{\alpha}=m_{1} x_{1}^{(\alpha)}+m_{2} x_{2}^{(\alpha)}+\cdots+m_{n} x_{n}^{(\alpha)} .
$$

Hence, if $x_{1}, x_{2}, \cdots, x_{n}$ are defined in the natural way, then

$$
x=m_{1} x_{1}+m_{2} x_{2}+\cdots+m_{n} x_{n} \in I\left(\prod_{\alpha \in B} D_{\alpha}\right) .
$$

Hence $\mathscr{D}$ is closed under direct products.

THEOREM 4.4. Let $(\mathscr{C}, \mathscr{T}),(\mathscr{T}, \mathscr{F})$ be a TTF theory such that $(\mathscr{T}, \mathscr{F})$ is splitting. Suppose the minimal ideal I in the filter $F(\mathscr{T})$ contains no nonzero nilpotent left ideals of $R$. Then $(\mathscr{C}, \mathscr{T})$ is central splitting if and only if $I$ is finitely generated as a right ideal.

Proof. $(\Leftarrow)$ : Since $(\mathscr{T}, \mathscr{F})$ is splitting, $R=\mathscr{T}(R) \oplus F$ with 
$F \in \mathscr{F}$. Then $R / F \in \mathscr{T}$, and hence $F \supseteqq I$ by the definition of $I$. By Lemma 4.3 the class $\mathscr{D}=\left\{D \in_{R} \mathscr{K} \mid I D=D\right\}$ is closed under products.

Claim $\mathscr{C}=\mathscr{D}$. Suppose $D \in \mathscr{D}$ and $\varphi: D \rightarrow T \in \mathscr{T}$. Then $\varphi(D)=$ $\varphi(I D)=I \cdot \varphi(D) \cong I \cdot T=0$ and so it follows that $\operatorname{Hom}(D, T)=0$ for all $T \in \mathscr{T}$. Thus $\mathscr{D} \subseteq \mathscr{C}$. Conversely, let $A \in \mathscr{C}$ and observe that $A / I A \in \mathscr{T}$ by the fact that $\mathscr{T}=\left\{M \in_{R} \mathscr{M} \mid I M=0\right\}$. Since $\mathscr{C}$ is closed under homomorphic images and $\mathscr{C} \cap \mathscr{T}=0$, it follows that $I A=A$. Thus $\mathscr{C} \cong \mathscr{D}$.

Next observe $I$ is essential in $F$. For if $K$ is a left ideal of $R$ contained in $F$ and $K \cap I=0$, then $I K=0$. Thus $K \subseteq \mathscr{T}(R) \cap F=0$.

Claim $x I \neq 0$ for all $0 \neq x \in F$. For if not, let $y I=0$ for $0 \neq y \in F$. Then $R y \cap I \neq 0$ since $I$ is essential in $F$. But $(R y \cap I)^{2} \subseteq$ $R y I=0$, which contradicts the hypothesis that $I$ contains no nonzero nilpotent left ideals.

Hence $F$ can be embedded as a left $R$-module in a product of copies of $I$ in the usual way. Moreover, $\prod_{\alpha \in A} I_{\alpha} \in \mathscr{D}$ (where $I_{\alpha}=I$ and $A$ is any index set) by Lemma 4.3 and the fact that $I^{2}=I$. Since $(\mathscr{T}, \mathscr{F})$ splits, $\mathscr{T}$ is closed under taking injective envelopes by Lemma 1.1. So [5], Theorem 2.9, gives $\mathscr{C}=\mathscr{D}$ is closed under submodules; in particular, $F \in \mathscr{C}$ and $F=I F=I$. But $I=\mathscr{C}(R)$, and hence $R=\mathscr{T}(R) \oplus F=\mathscr{T}(R)+\mathscr{C}(R)$ (ring direct sum). Hence, $(\mathscr{C}, \mathscr{T})$ is central splitting by Theorem 4.1.

$(\Leftrightarrow)$ : By Theorem 4.1, $R=\mathscr{C}(R)+\mathscr{T}(R)$ (ring direct sum) and hence $I=\mathscr{C}(R)$ is a principal right ideal.

Proposition 4.5. Let $(\mathscr{C}, \mathscr{T}),(\mathscr{T}, \mathscr{F})$ be a TTF theory such that $(\mathscr{T}, \mathscr{F})$ splits. Then the following are equivalent:

(1) $(\mathscr{C}, \mathscr{T})$ is central splitting.

(2) $(\mathscr{C}, \mathscr{T})$ is splitting.

(3) $\mathscr{C}$ is closed under taking injective envelopes.

(4) $\mathscr{C} \supseteq \mathscr{F}$.

Proof. $\quad(1) \Rightarrow(2)$ is trivial, and $(2) \Rightarrow$ (3) follows from Lemma 1.1.

$(3) \Rightarrow(4): \operatorname{By~}(\mathscr{T}, \mathscr{F})$ is splitting, Lemma 1.1, and [5], Theorem 2.9, $\mathscr{C}$ is closed under taking submodules. Let $F \in \mathscr{F}$ and note $\mathscr{C}(F) \subseteq F \leqq E(\mathscr{C}(F))$ : For if not, then there exists $0 \neq T \in \mathscr{T}$ such that $T \subseteq F$, which leads to a contradiction of $\mathscr{T} \cap \mathscr{F}=0$. But (3) and $\mathscr{C}$ closed under submodules then yield $F \in \mathscr{C}$, and hence $\mathscr{C} \supseteqq \mathscr{F}$.

$(4) \Rightarrow(1)$ : Since $(\mathscr{T}, \mathscr{F})$ is splitting, write $R=\mathscr{T}(R) \oplus F$ with $F \in \mathscr{F}$. Since $R / F \in \mathscr{T}$, then $F \supseteqq \mathscr{C}(R)$. But $F \in \mathscr{C}$ by (4), so $F=\mathscr{C}(R)$. Hence $R=\mathscr{T}(R)+\mathscr{C}(R)$ (ring direct sum), so that $(\mathscr{C}, \mathscr{T})$ is central splitting by Theorem 4.1 .

ExAmple 4.6. $\mathscr{T}$ is a TTF class and $(\mathscr{C}, \mathscr{T})$ is splitting, but 
not central splitting. Let $R$ be the ring of all $2 \times 2$ upper triangular matrices over the field $Q$ of rational numbers. Let $I=\left\{\left(\begin{array}{ll}0 & x \\ 0 & y\end{array}\right) \mid x, y \in Q\right\}$, so that $I$ is a two-sided idempotent ideal of $R$. Define:

$$
\begin{aligned}
& \mathscr{T}=\left\{M \in_{R} \mathscr{C} \mid I M=0\right\} \\
& \mathscr{F}=\left\{M \in_{R} \mathscr{M} \mid \operatorname{Hom}(T, M)=0 \text { for all } T \in \mathscr{T}\right\} \\
& \mathscr{C}=\left\{M \in_{R} \mathscr{C l} \mid \operatorname{Hom}(M, T)=0 \text { for all } T \in \mathscr{T}\right\} .
\end{aligned}
$$

Then $\mathscr{T}$ is a TTF class, and $(\mathscr{C}, \mathscr{T})$ and $(\mathscr{T}, \mathscr{F})$ are torsion theories. Since $R / I$ is a projective simple $R$-module, it follows that all modules in $\mathscr{T}$ are projective. Hence $(\mathscr{C}, \mathscr{T})$ is splitting. But $\mathscr{T}(R)=\left\{\left(\begin{array}{ll}x & y \\ 0 & 0\end{array}\right) \mid x, y \in Q\right\}$ is not a direct summand of $R$; so $(\mathscr{T}, \mathscr{F})$ is not splitting.

Proposition 4.7. Let $\mathscr{T}$ be a TTF class, and let $(\mathscr{C}, \mathscr{T})$ be a splitting torsion theory. Then the following are equivalent:

(1) $(\mathscr{C}, \mathscr{T})$ is central splitting

(2) $(\mathscr{C}, \mathscr{T})$ is hereditary

(3) $\mathscr{C}(R) \cap \mathscr{T}(R)=0$

(4) $\mathscr{C}(R) \cap \mathscr{T}(R)$ contains no nonzero nilpotent left ideals of $R$.

Proof. $\quad(1) \Rightarrow(2)$ is immediate from Theorem 4.1 (3).

If (2) holds, then $\mathscr{C}(R) \cap \mathscr{T}(R) \in \mathscr{C} \cap \mathscr{T}=0$, and hence (2) $\Rightarrow(3)$. $(3) \Rightarrow(4)$ is trivial.

Suppose (4) holds. Since $(\mathscr{C}, \mathscr{T})$ is splitting, $R=\mathscr{C}(R) \oplus T$ with $T \in \mathscr{T}$. Hence $\mathscr{T}(R) \supseteqq T$. But then

$$
[\mathscr{C}(R) \cap \mathscr{T}(R)]^{2} \subseteq \mathscr{C}(R) \cdot \mathscr{T}(R)=0
$$

implies $\mathscr{C}(R) \cap \mathscr{T}(R)=0$ by (4). Hence $\mathrm{T}=\mathscr{T}(R)$, and thus (1) holds by Theorem 4.1 (2).

\section{REFERENCES}

1. J. S. Alin and S. E. Dickson, Goldie's torsion theory and its derived functor, Pacific, J. Math. 24 (1968), 195-203.

2. H. Bass, Finitistic dimension and a homological generalization of semi-primary rings, Trans. Amer. Math. Soc. 90 (1960), 466-488.

3. R. L. Bernhardt, Splitting hereditary torsion theories over semi-perfect rings, Proc. Amer. Math. Soc. 22 (1969), 681-687.

4. V. Cateforis and F. Sandomierski, The singular submodule splits off, J. Algebra 10 (1968), 149-165.

5. S. E. Dickson, A torsion theory for abelian categories, Trans. Amer. Math. Soc. 121 (1966), 223-235.

6. - Direct decompositions of radicals, Proc. Conference on Categorical Algebra, La Jolla, 1965. 
7. - Noetherian splitting rings are Artinian, J. London Math. Soc. 42 (1967), $732-736$.

8. P. Freyd, Abelian categories, Harper and Row, New York, 1964.

9. J. Fuelberth, On commutative splitting rings, Proc. London Math. Soc. 20 (1970), 393-408.

10. P. Gabriel, Des catégories Abéliennes, Bull. Soc. Math. France 90 (1962), 323-448.

11. A. W. Goldie, Torsion free modules and rings, J. Algebra 1 (1964), 268-287.

12. R. M. Hamsher, Commutative rings over which every module has a maximal submodule, Proc. Amer. Math. Soc. 18 (1967), 1133-1137.

13. J. P. Jans, Some aspects of torsion, Pacific J. Math. 15 (1965), 1249-1259.

14. M. L. Teply, Some aspects of Goldie's torsion theory, Pacific J. Math. 29 (1969), 447-457.

15. E. A. Walker and C. Walker, Quotient categories and rings of quotients (to appear)

Received February 20, 1969.

UNIVERSITY OF FLORIDA

Gainesville, Florida 



\section{PACIFIC JOURNAL OF MATHEMATICS}

EDITORS

H. SAMELSON

Stanford University

Stanford, California 94305

RichaRd PIERCE

University of Washington

Seattle, Washington 98105
J. DUGUNDJI

Department of Mathematics

University of Southern California

Los Angeles, California 90007

RICHARD ARENS

University of California

Los Angeles, California 90024

\section{ASSOCIATE EDITORS}

E. F. BECKENBACH

B. H. NeumanN

F. WOLF

K. YOSHIDA

\section{SUPPORTING INSTITUTIONS}

UNIVERSITY OF BRITISH COLUMBIA

CALIFORNIA INSTITUTE OF TECHNOLOGY

UNIVERSITY OF CALIFORNIA

MONTANA STATE UNIVERSITY

UNIVERSITY OF NEVADA

NEW MEXICO STATE UNIVERSITY

OREGON STATE UNIVERSITY

UNIVERSITY OF OREGON

OSAKA UNIVERSITY

UNIVERSITY OF SOUTHERN CALIFORNIA
STANFORD UNIVERSITY

UNIVERSITY OF TOKYO

UNIVERSITY OF UTAH

WASHINGTON STATE UNIVERSITY

UNIVERSITY OF WASHINGTON

AMERICAN MATHEMATICAL SOCIETY CHEVRON RESEARCH CORPORATION TRW SYSTEMS

NAVAL WEAPONS CENTER 


\section{Pacific Journal of Mathematics}

May, 1970

Johan Aarnes, Edward George Effros and Ole A. Nielsen, Locally compact

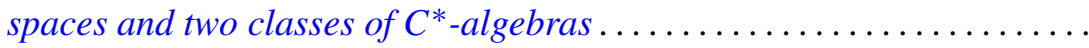

Allan C. Cochran, R. Keown and C. R. Williams, On a class of topological

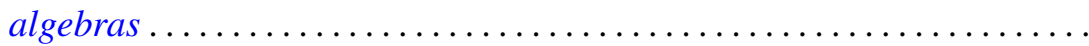

John Dauns, Integral domains that are not embeddable in division rings ....

Robert Jay Daverman, On the number of nonpiercing points in certain

crumpled cubes.....................................

Bryce L. Elkins, Characterization of separable ideals ................

Zbigniew Fiedorowicz, A comparison of two naturally arising uniformities

on a class of pseudo-PM spaces ...........................

Henry Charles Finlayson, Approximation of Wiener integrals of functionals

continuous in the uniform topology ........................

Theodore William Gamelin, Localization of the corona problem ...........

Alfred Gray and Paul Stephen Green, Sphere transitive structures and the

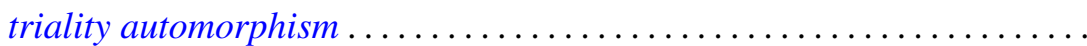

Charles Lemuel Hagopian, On generalized forms of aposyndesis ..........

J. Jakubík, On subgroups of a pseudo lattice ordered group ...............

Cornelius W. Onneweer, On uniform convergence for Walsh-Fourier

series..................................

Stanley Joel Osher, On certain Toeplitz operators in two variables ...

Washek (Vaclav) Frantisek Pfeffer and John Benson Wilbur, On the

measurability of Perron integrable functions............

Frank J. Polansky, On the conformal mapping of variable regions...

Kouei Sekigawa and Shûkichi Tanno, Sufficient conditions for a Riemannian manifold to be locally symmetric ...................

James Wilson Stepp, Locally compact Clifford semigroups ....

Ernest Lester Stitzinger, Frattini subalgebras of a class of solvable Lie

algebras ................................

George Szeto, The group character and split group algebras...

Mark Lawrence Teply, Homological dimension and splitting torsion

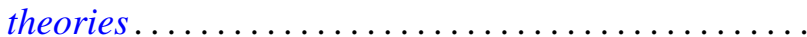

David Bertram Wales, Finite linear groups of degree seven. II ...

Robert Breckenridge Warfield, Jr., An isomorphic refinement theorem for

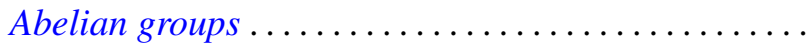

James Edward West, The ambient homeomorphy of an incomplete subspace

of infinite-dimensional Hilbert spaces................

Peter Wilker, Adjoint product and hom functors in general topology ...

Daniel Eliot Wulbert, A note on the characterization of conditional 